\title{
含不同取代吲哚修饰的方酸菁衍生物的合成及光学性能的研究
}

\author{
张碧玮袁展翔徐建兴籍少敏* 霍延平* 梁 亮* \\ (广东工业大学轻工化工学院 广州 510006)
}

\begin{abstract}
摘要 通过缩合反应合成了四个含不同取代吲槑修饰的方酸菁衍生物(SQ、DISQ、MeSQ、DIMeSQ). 方酸菁衍生物 是一种 D-A-D 构型的染料，由方酸与两个富电子基团通过共轭连接而成. 化合物结构通过 ${ }^{1} \mathrm{H} \mathrm{NMR},{ }^{13} \mathrm{C} N \mathrm{NMR}$ 和高分辨 质谱进行表征. 同时, 对于它们的紫外-可见吸收、菼光发射、苂光量子产率、苂光寿命及单线态氧量子产率等光物理 性质也做了研究. 结果显示, 在二氯甲烷溶液中, SQ、DISQ、MeSQ 和 DIMeSQ 的吸收波长在 $600 \sim 700 \mathrm{~nm}$, 发射波 在 620 $800 \mathrm{~nm}$, 均在近红外区域. 在应用方面, 4 个方酸菁衍生物敏化单线态氧性能被研究, 其中 DISQ 获得最高的单 线态氧量子产率 $(6 \%)$, 是 DIMeSQ 的 2 倍(3\%), 可能因为 $N$-氢取代分子相比 $N$-甲基取代分子, 更有利于分子的系间㡍 跃. 结合单晶结构和理论计算证明分子内 $\mathrm{N}-\mathrm{H} \cdots \mathrm{O}$ 作用力有利于分子的系间䇀跃, 表明分子 DISQ 可以作为光动力治 疗的潜在药物.
\end{abstract}

关键词 方酸菁衍生物; 三重态; 单线态氧; 系间鼌跃

\section{Synthesis and Optical Properties of Different Substituted- Indole-Modified Squaraine Derivatives}

\author{
Zhang, Biwei \\ Yuan, Zhanxiang \\ $\mathrm{Xu}$, Jianxing \\ $\mathrm{Ji}$, Shaomin* \\ Huo, Yanping* \\ Liang, Liang* \\ (School of Chemicals Engineering and Light Industry, Guangdong University of Technology, Guangzhou 510006)
}

\begin{abstract}
Four squaraine derivatives SQ, DISQ, MeSQ and DIMeSQ with different substitution indole modifications were synthesized through condensation reaction. Squaraine derivative is one kind of D-A-D configuration dyes, which is formed by connecting squaraine with two electron-rich groups through conjugation. The squaraine derivatives were characterized by ${ }^{1} \mathrm{H}$ NMR, ${ }^{13} \mathrm{C}$ NMR and high resolution mass spectrometry. The photophysical properties of these squaraine derivatives were studied, such as UV-visible absorption, fluorescence emission, fluorescence quantum yield, fluorescence lifetimes, singlet oxygen quantum yields, and so on. The results showed that, SQ, DISQ, MeSQ and DIMeSQ showed stronger absorption band at $600 \sim 700 \mathrm{~nm}$ and emission band at $620 \sim 800 \mathrm{~nm}$ in dichloromethane, which were in near-infrared filed. These four squaraine derivatives were used for singlet oxygen generation, and the highest singlet oxygen quantum yield $(6 \%)$ was observed for DISQ, which is twice as high as that of DIMeSQ (3\%). It is proposed that iodine-substituted and $N$-hydrogensubstituted molecules are more conducive to intersystem crossing than $N$-methyl substituted molecules. In addition, It is confirmed that the intramolecular $\mathrm{N}-\mathrm{H} \cdots \mathrm{O}$ force is beneficial to the intersystem crossing of molecules by combining single crystal structure and theoretical calculations. It showed that DISQ could be used as a potential drug for photodynamic therapy. Keywords squaraine derivatives; triplet state; singlet oxygen; intersystem crossing
\end{abstract}

近年来，有机染料，特别是近红外有机染料的开发 和应用得到了广泛的关注, 它们被应用于光电子器 件 ${ }^{[1]} 、$ 化学/生物传感 ${ }^{[2-3]} 、$ 生物探针 ${ }^{[4]}$ 及光动力治疗 ${ }^{[5-11]}$ 等领域的研究. 与无机苂光材料相比, 有机近红外染料 因为其良好的光物理性能，应用前景更加广泛。通常，
它们的强吸收主要集中在远红光和近红外区(780 1100 $\mathrm{nm})$ ，在生物样品的苂光检测中，可以避免蛋白质等的 自身散射和荧光背景干扰; 在肿瘤光动力治疗中, 可以 达到更深的组织穿透效果; 在多种溶剂, 特别是缓冲液 中有很好的吸收和发射, 有很强的光化学稳定性. 常见

\footnotetext{
* Corresponding authors. E-mail: smji@gdut.edu.cn; yphuo@gdut.edu.cn; liang1@gdut.edu.cn Received February 20, 2020; revised April 28, 2020; published online June 11, 2020.

Project supported by the National Natural Science Foundation of China (Nos. 21975053, 21975055). 国家自然科学基金(Nos. 21975053, 21975055)资助项目。
} 
的近红外苂光染料可以分为以下几类: 菁染料 ${ }^{[12-15]}$ 、含 四吡咯基团的近红外苂光染料 ${ }^{[16]}$ 、咕吨类荧光染料 ${ }^{[17]}$ 、 噻嗪类和嗯嗪类近红外苂光染料 ${ }^{[18]}$ 、二氟化嗍-二吡咯 甲烷荧光染料 ${ }^{[19-22]}$ 等.

方酸菁染料作为一类近红外菁染料, 自 1959 年 Cohen $^{[23]}$ 合成以来, 由于其独特的构型和优良的光化学 稳定性, 在理论研究和实际应用中一直备受关注. 方酸 菁染料具有共振稳定的两性离子结构, 是由方酸与两个 富电子部分缩合形成的供体-受体-供体(D-A-D)骨架结 构 ${ }^{[24]}$. 因此, 研究者们可以通过改变供体结构, 从而调 节方酸菁染料的光学性能.

由于吲哚基团相对其他供电子基团, 其摩尔消光系 数大、苂光性能良好、最大吸收波长可调谐范围大、分 子平面性更强、易于合成并且有相对较高的稳定 性 ${ }^{[25-26]}$; 此外, 吲哚中含有 $\mathrm{N}$ 元素, 可以进行支链修饰. 因此，我们设计合成了含不同取代吲哚修饰的 SQ、

DISQ、MeSQ 和 DIMeSQ 四个方酸菁衍生物. 如图 1 所示, 方酸菁衍生物通过在方酸母核 1,3-位引入吲哚结 构, 形成 D-A-D 构型; 然后在吲哚结构上引入不同的碘 取代、 $N$-氢取代或 $N$-甲基取代, 对比重原子效应和分子 内 $\mathrm{N}-\mathrm{H} \cdots \mathrm{O}$ 作用力对分子系间窝跃的影响. 通过测试 发现, 所有化合物在二氯甲烷溶液中均具有强的近红外 吸收性. 此外, 通过单线态氧量子产率测试发现, 分子 DISQ 可获得最高的单线态氧量子产率. 结合单晶结构 解析和理论计算证明, 分子内 $\mathrm{N}-\mathrm{H} \cdots \mathrm{O}$ 作用力更有利 于分子的系间窝跃, 表明分子 DISQ 可以作为光动力治 疗的潜在光敏剂.

\section{1 结果与讨论}

\subsection{D-A-D 型方酸菁衍生物的结构设计与合成策略}

对于有机光敏剂而言, 提高单线态氧产生效率的关 键在于提高分子单重态至三重态的系间窝跃. 而主要策 略集中在以下两点: 第一, 引入重原子以增强光敏剂自 旋-轨道耦合作用; 第二, 降低分子最低单重态 $\left(\mathrm{S}_{1}\right)$ 至最 低三重态 $\left(\mathrm{T}_{1}\right)$ 之间的能级差 $\left(\Delta E_{\mathrm{st}}\right)$. 如图 1 所示, 在分子 设计方面, 由于吲哚基团相对其他供电子基团, 具有摩 尔消光系数大、苂光性能良好、最大吸收波长可调谐范 围大、分子平面性强、易于合成和较高的稳定性等优 点 ${ }^{[25-26]}$; 而且, 吲哚中含有 $\mathrm{N}$ 元素, 可以进行支链修饰. 因此, 向方酸母核的 1,3-位引入吲哚结构, 然后在吲哚 结构上引入不同的碘取代、 $N$-氢取代或 $N$-甲基取代得到 1,3-对称型的方酸菁衍生物. 本工作分子设计的目的主 要有两点: 第一, 通过在吲哚结构上引入碘元素, 可以 形成重原子效应，以增强光敏剂自旋-轨道耦合作用， 从而增大 $\mathrm{S}_{0} \rightarrow \mathrm{T}_{1}$ 吸收跃迁和 $\mathrm{S}_{1} \rightarrow \mathrm{T}_{1}$ 体系间鼌跃的几率,
Components

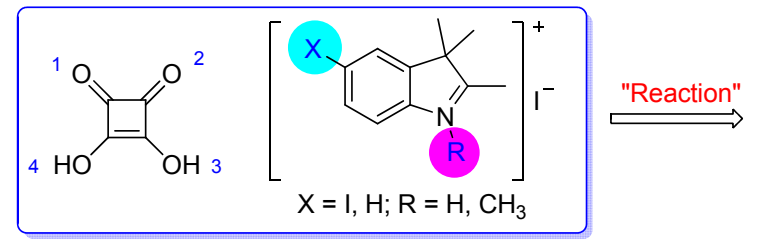

Integration

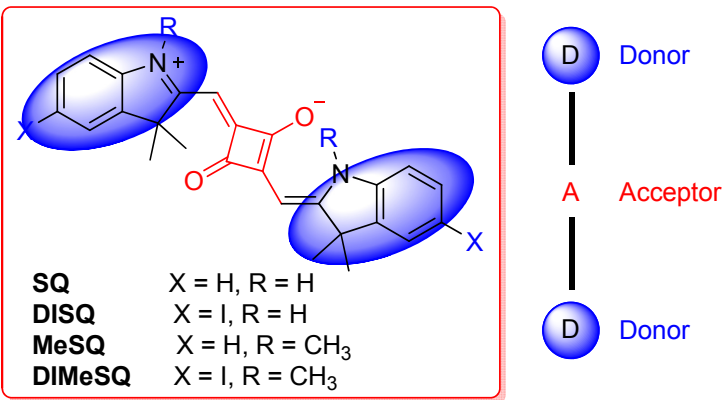

(1) Synthesized D-A-D type indole-modified squaraine derivatives through condensation

(2) Strong NIR-absorption in dichloromethane

(3) The heavy element I modifies the molecule, which is good for intersystem crossing

(4) Intramolecular $\mathrm{N}-\mathrm{H} \cdots \mathrm{O}$ force is beneficial to intersystem crossing

图 1 方酸菁衍生物的分子设计、结构关系以及主要影响因素 Figure 1 Molecular design, structural relationship and main influencing factors of squaraine derivatives

即增加了 $\mathrm{T}_{1}$ 态粒子的布局数，有利于促进分子系间鼌 跃; 第二, 在吲哚结构上引入 $\mathrm{N}-\mathrm{H}$ 取代, 可以形成分 子内 $\mathrm{N}-\mathrm{H} \cdots \mathrm{O}$ 作用力, 有利于稳定分子中心受体结构, 造成电荷分离程度减小，因而使 $\pi \rightarrow \pi^{*}$ 跃迁能级降低, 使其吸收带的最大吸收波长向长波方向移动, 有利于 $\mathrm{S}_{1}$ 态的形成，从而促进了分子的系间鼌跃. 在分子合成策 略方面, 如 Scheme 1 所示, 其主要步骤包括吲哚结构的 构筑和与方酸连接两部分. 第一部分吲哚结构的构筑主 要包括三步: 第一步, 将不同取代苯胺与亚硝酸钠在酸 性介质中进行重氮化反应, 生成重氮盐. 再用还原剂氯 化亚锡还原生成重氮苯磺酸盐, 经酸析, 生成苯肼盐酸 盐. 最后用碱液中和制得不同取代苯肼. 第二步, 通过 Fischer 吲哚合成法, 将不同取代苯肼与等当量的酮, 在 酸中加热、回流得到苯腙. 其在酸催化下进行重排、消 除氨, 从而得到吲哚化合物. 第三步, 采用碘甲烷作为 甲基化试剂得到甲基化吲哚. 第二部分与方酸连接，是 以方酸和两倍物质的量的不同取代吲哚作为反应物，正 丁醇和甲苯作为反应溶剂，在回流条件下，用 Deanstark 分水器分离出反应中生成的水. 随着反应的不断 进行，反应溶液颜色逐渐变深，反应完成时，产物会在 反应液中析出，得到四个具有近红外吸收的含不同取代 吲哚修饰的方酸菁衍生物(SQ、DISQ、MeSQ、DIMeSQ). 中间体 2 按照文献[27]中的方法制备，最终产物和中间 体 3、4 按照文献[28]中的方法制备，最终产物通过核磁 
共振与质谱进行表征.

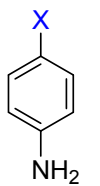

$1 b X=1$
(1) $\mathrm{HCl}, \mathrm{NaNO}_{2}$ $0{ }^{\circ} \mathrm{C}, 90 \mathrm{~min}$

(2) $\mathrm{SnCl}_{2}$, concd. $\mathrm{HCl}$ $0^{\circ} \mathrm{C}, 60 \mathrm{~min}$

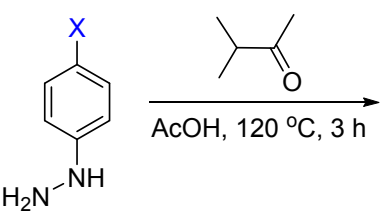

2b $X=1,66 \%$ yied<smiles>[X]c1ccc2c(c1)C(C)(C)C(C)=N2</smiles>

$3 \mathrm{a} X=\mathrm{H}, 70 \%$ yied 3b $X=1,79 \%$ yied<smiles></smiles>
4

RI, microwave $135^{\circ} \mathrm{C}, 20 \mathrm{~min}$

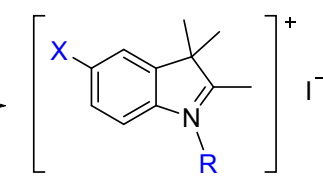

4a $\mathrm{X}=\mathrm{H}, \mathrm{R}=\mathrm{CH}_{3}, 48 \%$ yield 4b $X=I, R=\mathrm{CH}_{3}, 57 \%$ yield

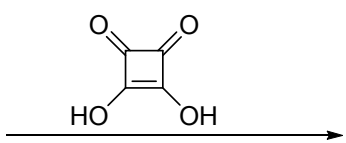

n-butanol/toluene $115^{\circ} \mathrm{C}$, refluex, $4 \sim 5 \mathrm{~h}$ 3a $X=H, R=H ; 3 b X=I, R=H$

4a $X=\mathrm{H}, \mathrm{R}=\mathrm{CH}_{3} ; \mathbf{4 b} X=\mathrm{I}, \mathrm{R}=\mathrm{CH}_{3}$

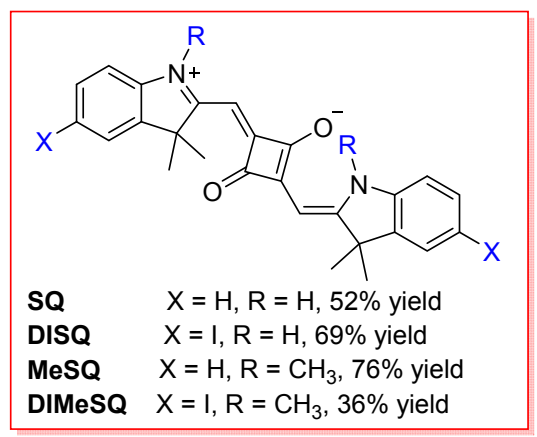

图式 1 目标化合物 SQ、DISQ、MeSQ 和 DIMeSQ 的合成 Scheme 1 Synthesis of target compounds SQ、DISQ、MeSQ and DIMeSQ

\section{2 紫外-可见吸收与荧光发射光谱研究}

首先, 我们对化合物的吸收和发射光谱进行了测 试. 通过吸收光谱(图 2a 和表 1)可以看出, 所有化合物 在 $360 \mathrm{~nm}$ 处均有一个弱的吸收, 这是典型的吲哚吸收 谱带; 在 525 720 nm 之间具有一个强吸收带, 是典型 的方酸菁染料吸收谱带. 分子 SQ、DISQ、MeSQ 和 DIMeSQ 的最大吸收波长分别是 655、666、633 和 644 $\mathrm{nm}$, 所对应的摩尔消光系数分别为 $2.13 \times 10^{5} 、 1.80 \times$ $10^{5} 、 1.80 \times 10^{5}$ 和 $1.78 \times 10^{5} \mathrm{~mol} \cdot \mathrm{L}^{-1} \cdot \mathrm{cm}^{-1}$, 由此可以证 明所有化合物均具有强的近红外吸收性能. 其中分子 DISQ 和 DIMeSQ 与分子 SQ 和 MeSQ 相比, 最大吸收 峰都红移了 $11 \mathrm{~nm}$, 说明碘取代对激发态性能起到一定 的调控作用, 能促进吸收波长红移. 分子 SQ 和 DISQ 与 MeSQ 和 DIMeSQ 相比, 最大吸收峰也都红移了 $22 \mathrm{~nm}$, 说明 $N$-氢取代相对于 $N$-甲基取代, 由于分子内形成
$\mathrm{N}-\mathrm{H} \cdots \mathrm{O}$ 作用力，稳定了分子受体 $\mathbf{A}$; 同时，密度泛函 理论计算结果显示, $\mathrm{N}-\mathrm{H} \cdots \mathrm{O}$ 作用增加了分子平面性, 造成分子电荷分离程度减小, 从而使 $\pi \rightarrow \pi *$ 跃迁能级降 低, 吸收带的最大吸收波长向长波方向移动. 由此可见, $N$-氢取代或碘取代均能明显促进吸收波长红移，有益于 分子对光的捕获.
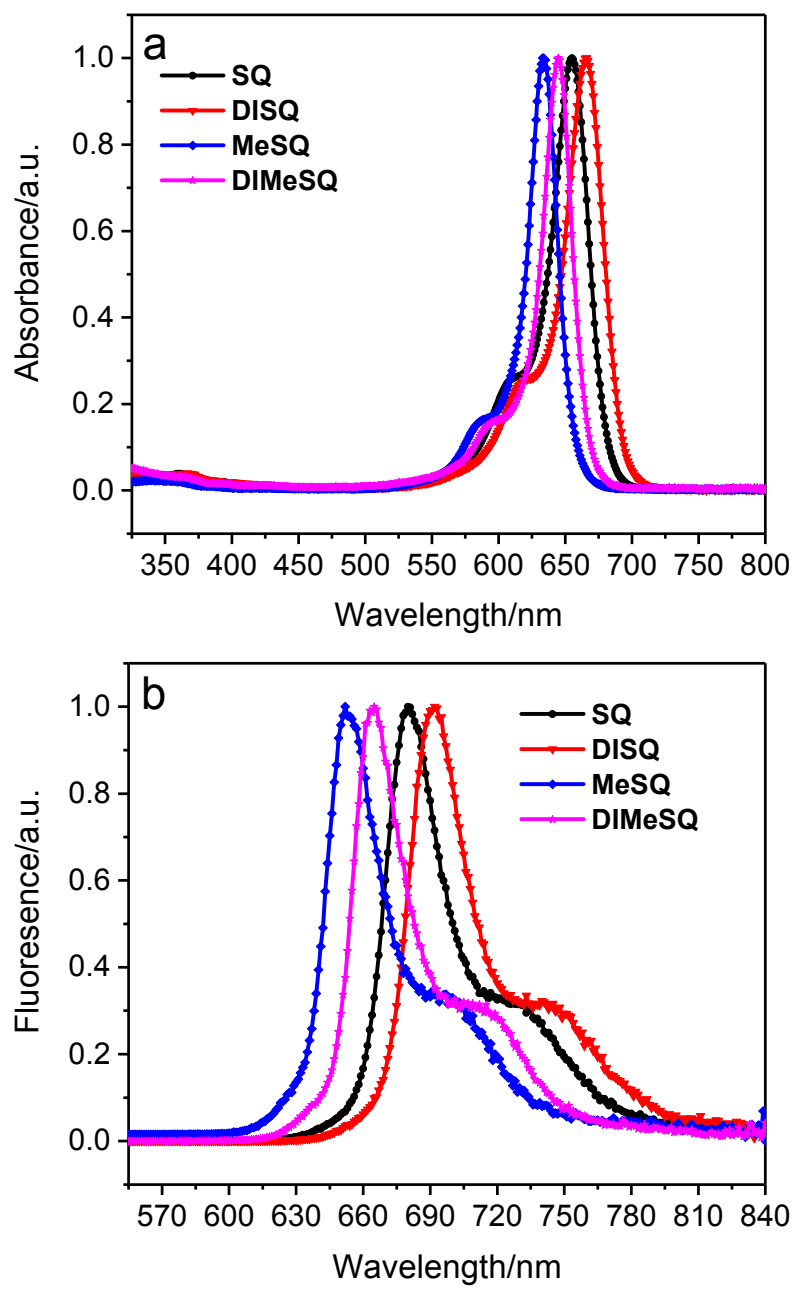

图 2 分子在二氯甲烷中的(a)紫外-可见吸收光谱与(b)苂光发 射光谱

Figure 2 (a) UV-Vis absorption spectra and (b) fluorescence emission spectra of compounds in dichloromethane $c=1.0 \times 10^{-5} \mathrm{~mol} \cdot \mathrm{L}^{-1}$

从它们的荧光发射光谱来看(图 $2 \mathrm{~b}$ ), 所有化合物的 荧光发射光谱都有相似的形状, 而且发射波谱与吸收波 谱呈镜像关系, 它们均具有较小的 Stokes 位移, 分别是 25、30、26 和 $20 \mathrm{~nm}$, 这说明光激发过程中, 分子结构 变化是可以忽略的. 其中分子 DISQ 与 SQ 相比最大发 射峰红移了 $16 \mathrm{~nm}$, 分子 DIMeSQ 与 MeSQ 相比最大发 射峰红移了 $5 \mathrm{~nm}$, 说明碘取代能对基态作用, 使发射波 长红移. 分子 SQ 相对 MeSQ 最大发射峰红移了 $21 \mathrm{~nm}$, 分子 DISQ 相对 DIMeSQ 最大发射峰红移了 $32 \mathrm{~nm}$, 同 
表 1 化合物的基本光物理性能

Table 1 Photophysical parameters of compounds

\begin{tabular}{|c|c|c|c|c|c|c|c|}
\hline Compd. & $\lambda_{\mathrm{abs}}{ }^{a} / \mathrm{nm}$ & $\lambda_{\mathrm{abs}}{ }^{b} / \mathrm{nm}$ & $\Delta v^{c} /\left(\mathrm{nm} / \mathrm{cm}^{-1}\right)$ & $\varepsilon^{d} /\left(10^{5} \mathrm{~L} \cdot \mathrm{mol}^{-1} \cdot \mathrm{cm}^{-1}\right)$ & $\Phi_{\mathrm{f}}{ }^{e} / \%$ & $\tau^{f} / \mathrm{ns}$ & $\Phi_{\Delta}^{g} / \%$ \\
\hline SQ & 360,655 & 680 & $25 / 561$ & 2.13 & 42 & 3.44 & 4 \\
\hline DISQ & 363,666 & 696 & $30 / 647$ & 1.80 & 23 & 1.12 & 6 \\
\hline MeSQ & 361,633 & 659 & $26 / 623$ & 1.80 & 50 & 3.75 & 2 \\
\hline DIMeSQ & 361,644 & 664 & $20 / 467$ & 1.78 & 31 & 1.46 & 3 \\
\hline
\end{tabular}

${ }^{a}$ The maximum absorption wavelength; ${ }^{b}$ the maximum emission wavelength; ${ }^{c}$ Stokes shift; ${ }^{d}$ molar extinction coefficient; ${ }^{e}$ fluorescence quantum yield; ${ }^{f}$ fluorescence lifetime; ${ }^{g}$ singlet oxygen quantum yield.

样说明由于氢键的作用，使激发态和基态溶剂重组很 小, 即分子在溶液中容易形成聚集态, 意味着分子 $\mathrm{S}_{1} \rightarrow \mathrm{S}_{0}$ 过程中, 溶剂的驰豫时间较长, 分子基态能级不 稳定性增加, 从而表现出发射光谱的红移. 因此, $N$-氢 取代或碘取代均能明显促进发射光谱红移.

\section{3 荧光量子产率 $\Phi_{\mathrm{f}}$ 与荧光寿命 $\tau$}

苂光量子产率 $\Phi_{\mathrm{f}}$ 与苂光寿命 $\tau$ 测试结果均见表 1 所示, 分子 SQ、DISQ、MeSQ 和 DIMeSQ 的苂光量子 产率 $\Phi_{\mathrm{f}}$ 在 $20 \% \sim 50 \%$ 之间，分别是 $42 \% 、 23 \% 、 50 \%$ 和 $31 \%$. 其中分子 DISQ 与 SQ 相比苂光量子产率 $\Phi_{\mathrm{f}}$ 降低 了 $19 \%$, DIMeSQ 与 MeSQ 相比苂光量子产率 $\Phi_{\mathrm{f}}$ 降低了 $19 \%$, 说明碘取代对荧光量子产率 $\Phi_{\mathrm{f}}$ 的影响很大, 表明 碘取代有利于方酸分子的系间窝跃. 分子 SQ 和 DISQ 相对分子 MeSQ 和 DIMeSQ, 苂光量子产率 $\Phi_{\mathrm{f}}$ 均降低 了 $8 \%$, 说明由于氢键作用的影响, 苂光量子产率 $\Phi_{\mathrm{f}}$ 减 小. 证明碘取代和 $N$-氢取代分子相比 $N$-甲基取代分子 更有利于分子的系间窝跃.

通过测试苂光寿命(图 3) 可以得到, 分子 SQ、 DISQ、MeSQ 和 DIMeSQ 的荧光寿命 $\tau$ 分别为 3.44、 1.12、3.75 和 $1.46 \mathrm{~ns}$. 分子 SQ 对比 DISQ 苂光寿命 $\tau$ 长了 $2.32 \mathrm{~ns}$, 分子 MeSQ 对比 DIMeSQ 苂光寿命 $\tau$ 长了 $2.29 \mathrm{~ns}$, 这与苂光量子产率测试结果相对应, 进一步证 明了, 碘取代有利于增大方酸分子的 $\mathrm{S}_{0} \rightarrow \mathrm{T}_{1}$ 吸收跃迁和 $\mathrm{S}_{1} \rightarrow \mathrm{T}_{1}$ 体系间䇀跃的几率.

\section{4 敏化单线态氧}

众所周知, 三重态光敏剂可以敏化氧气产生单线态 氧, 从而杀死癌细胞或肿瘤组织 ${ }^{[5-11]}$. 我们利用 1,3 -二苯 基异苯并呋喃作为单线态氧捕获剂, 测试了分子 $\mathbf{S Q}$ 、

DISQ、MeSQ 和 DIMeSQ 的敏化单线态氧能力(图 4). 测试发现，分子 DISQ 随着光照时间的增加, 1,3-二苯基 异苯并呋喃 $415 \mathrm{~nm}$ 处的吸光度降低最为明显. 通过公 式计算出它们在二氯甲烷溶液中的单线态氧量子产率 $\Phi_{\Delta}$ 分别为 $4 \% 、 6 \% 、 2 \%$ 和 $3 \%$. 分子 DISQ 和 DIMeSQ 与分子 SQ 和 MeSQ 相比, 单线态氧量子产率 $\Phi_{\Delta}$ 分别 提高了 $2 \%$ 和 $1 \%$. 进一步暗示碘取代增强了方酸分子自 旋一轨道耦合作用, 从而增大了 $\mathrm{S}_{0} \rightarrow \mathrm{T}_{1}$ 吸收跃迁和 $\mathrm{S}_{1} \rightarrow$
$\mathrm{T}_{1}$ 体系间窝跃的几率，即增加了 $T_{1}$ 态粒子的布局数，有

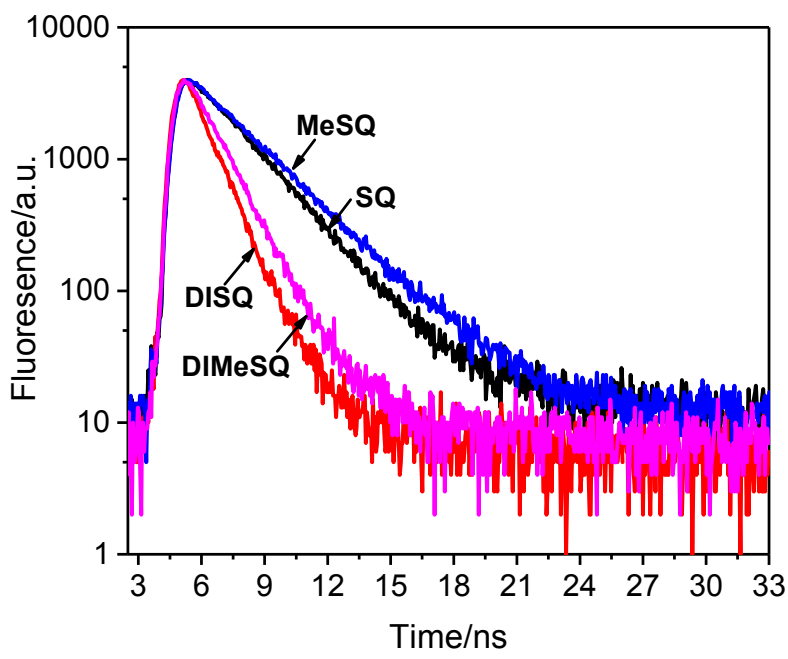

图 3 分子在二氯甲烷中的苂光寿命图

Figure 3 Fluorescence lifetime spectrum of the compounds in dichloromethane

$$
c=1.0 \times 10^{-5} \mathrm{~mol} \cdot \mathrm{L}^{-1}
$$

利于促进分子系间窝跃. 其中分子 DISQ 的单线态氧量 子产率是 DIMeSQ 的 2 倍, 可能由于分子内 $\mathrm{N}-\mathrm{H} \cdots \mathrm{O}$ 和重元素碘共同作用的结果. 通过对比 Danaboyina 等 ${ }^{[29]}$ 文献中报道的苯修饰一方酸化合物的单线态氧量子 产率 $(<0.1 \%)$, 进一步说明我们分子设计的方法是可行 的. 我们考虑在之后的研究中, 对分子 DISQ 进行进一 步肿瘤靶向基团修饰, 并且采用纳秒瞬态吸收光谱进行 三重态寿命测试和飞秒瞬态吸收光谱测试能量转化过 程, 确定分子 DISQ 可以作为光动力治疗的潜在药物.

\section{5 晶体结构的描述}

单晶 $\mathrm{X}$ 射线衍射数据表明，化合物 DISQ 为中性， 属于单斜晶系, 空间群是 $P 21 、 Z=2$, 晶体结构参数列 于表 2(数据存于英国剑桥数据中心, $\mathrm{CCDC}$ 号为 1973449). 从图 5、图 6 可知，化合物 DISQ 的分子结构 中心轴对称, 且接近理想平面形结构. 其中分子内 $\mathrm{N}-$ $\mathrm{H} \cdots \mathrm{O}$ 距离为 $0.189 \mathrm{~nm}$, 与高斯计算结果, 分子 $\mathbf{S Q}$ 和 DISQ 分子内 $\mathrm{N}-\mathrm{H} \cdots \mathrm{O}$ 距离 $0.177 \mathrm{~nm}$ 相近, 进一步证 明分子内存在 $\mathrm{N}-\mathrm{H} \cdots \mathrm{O}$ 作用力, 这与理论计算和测试 结果一致. 

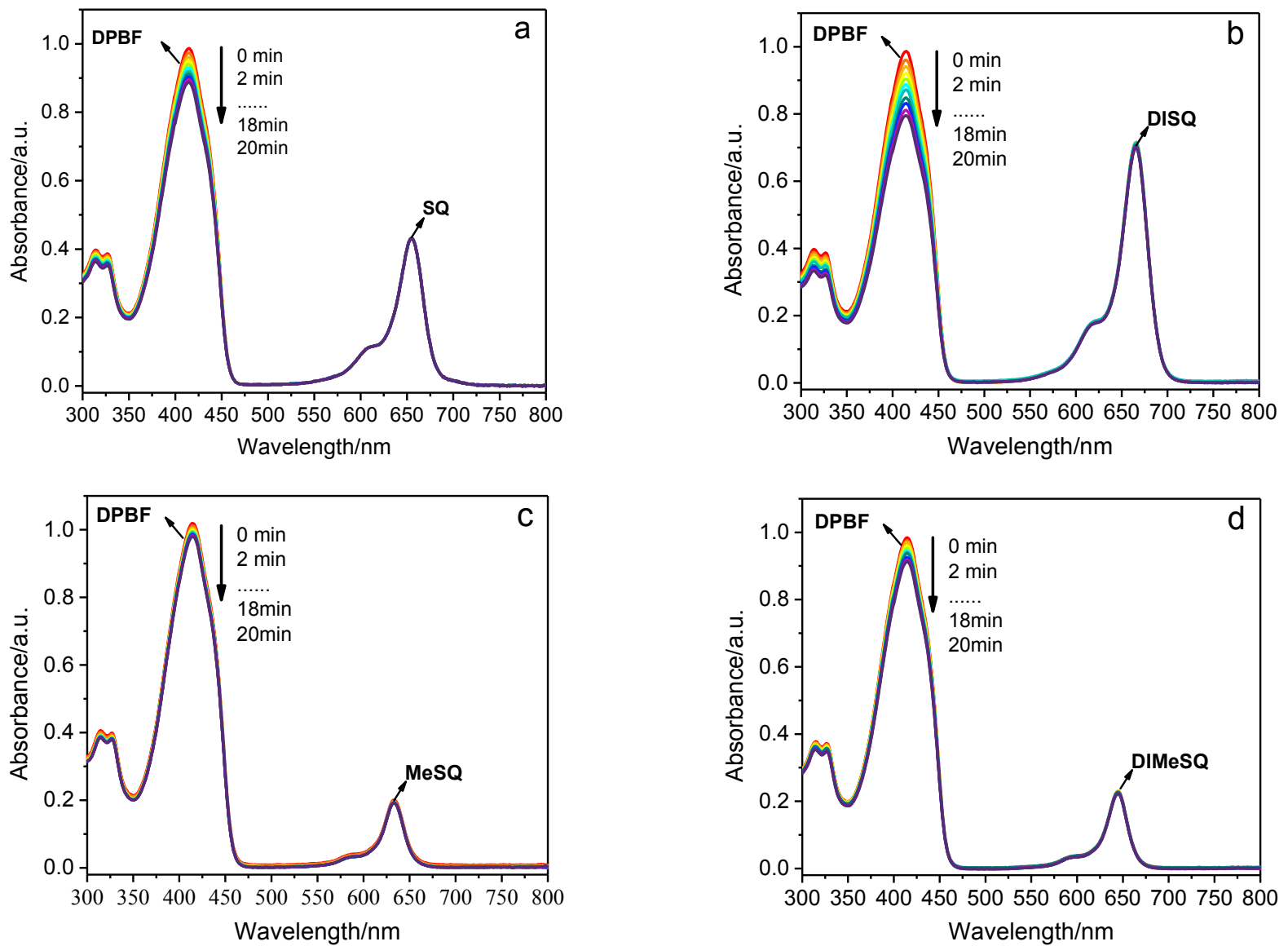

图 4 分子 SQ (a), DISQ (b), MeSQ (c)和 DIMeSQ (d) 在二氯甲烷中吸收光谱随时间变化

Figure 4 Absorption spectrum changes over time of molecules SQ (a), DISQ (b), MeSQ (c) and DIMeSQ (d) in dichloromethane $c=1.0 \times 10^{-5} \mathrm{~mol} \cdot \mathrm{L}^{-1}$

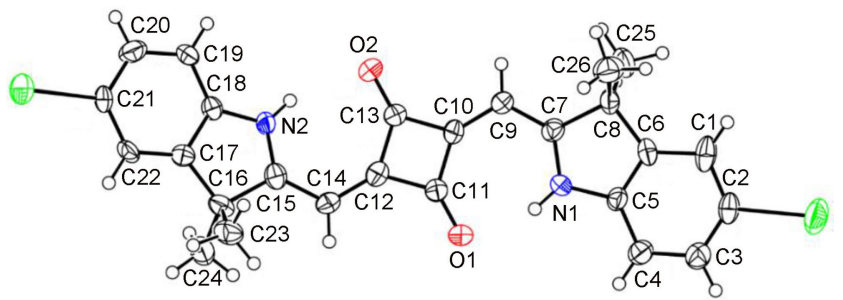

图 5 化合物 DISQ 的晶胞结构

Figure 5 Molecular structure of compound DISQ

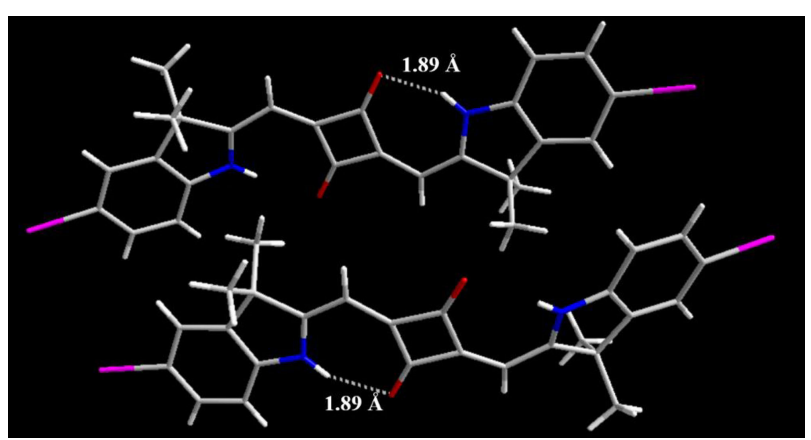

图 6 化合物 DISQ 的 $\mathrm{N}-\mathrm{H} \cdots \mathrm{O}$ 作用力(白色虚线)的二维图 形

Figure 6 Two-dimensional graph of the $\mathrm{N}-\mathrm{H} \cdots \mathrm{O}$ force (white dotted line) of the compound DISQ

\section{6 理论计算}

首先，通过密度泛函理论计算，对化合物 $S Q$ 、 DISQ、MeSQ 和 DIMeSQ 的构型进行了研究, 发现 4 个化合物都近似平面结构, 如图 7 所示. 其中分子 SQ

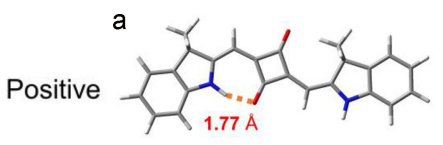

Side
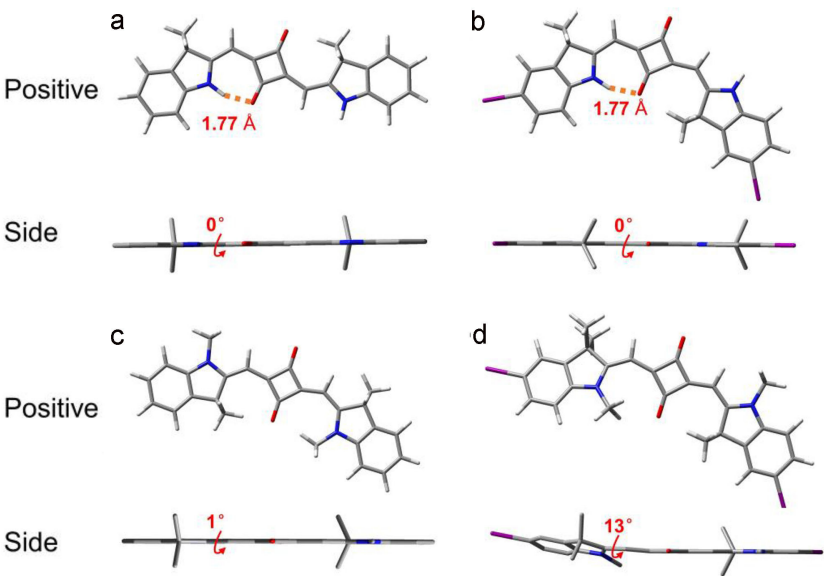

图 7 (a) SQ, (b) DISQ, (c) MeSQ 和(d) DIMeSQ 分子的优化 结构

Figure 7 Optimized structures of (a) SQ, (b) DISQ, (c) MeSQ, and (d) DIMeSQ 
表 2 化合物 DISQ 的 X 射线衍射数据

Table $2 \mathrm{X}$ ray diffraction data for compound DISQ

\begin{tabular}{|c|c|}
\hline Empirical formula & $\mathrm{C}_{26} \mathrm{H}_{22} \mathrm{I}_{2} \mathrm{~N}_{2} \mathrm{O}_{2}$ \\
\hline Formula weight & 648.25 \\
\hline Temperature/K & 293(2) \\
\hline Crystal system & triclinic \\
\hline Space group & $P-1$ \\
\hline$a / \mathrm{nm}$ & $0.98488(9)$ \\
\hline$b / \mathrm{nm}$ & $0.98904(10)$ \\
\hline$c / \mathrm{nm}$ & $1.46689(17)$ \\
\hline$\alpha /\left(^{\circ}\right)$ & $73.786(10)$ \\
\hline$\beta /\left(^{\circ}\right)$ & $77.375(9)$ \\
\hline$\gamma /\left({ }^{\circ}\right)$ & $63.135(10)$ \\
\hline Volume $/ \mathrm{nm}^{3}$ & $1.2168(2)$ \\
\hline$Z$ & 2 \\
\hline$\rho_{\text {calcd }} /\left(\mathrm{g} \cdot \mathrm{cm}^{-3}\right)$ & 1.769 \\
\hline$\mu / \mathrm{mm}^{-1}$ & 2.610 \\
\hline$F(000)$ & 628.0 \\
\hline Crystal size $/ \mathrm{mm}^{3}$ & $0.12 \times 0.11 \times 0.1$ \\
\hline Radiation & Mo $\mathrm{K} \alpha(\lambda=0.71073 \mathrm{~nm})$ \\
\hline $2 \theta$ range for data collection $/\left(^{\circ}\right)$ & 4.664 to 49.998 \\
\hline Index ranges & $\begin{array}{l}-11 \leqslant h \leqslant 8, \quad-11 \leqslant \mathrm{k} \leqslant 11, \\
-17 \leqslant 1 \leqslant 13\end{array}$ \\
\hline Reflections collected & 8092 \\
\hline Independent reflections & $\begin{array}{l}4276\left[R_{\text {int }}=0.0420,\right. \\
\left.R_{\text {sigma }}=0.0782\right]\end{array}$ \\
\hline Data/restraints/parameters & $4276 / 0 / 293$ \\
\hline Goodness-of-fit on $F^{2}$ & 1.151 \\
\hline Final $R$ indexes $[I \geqslant 2 \sigma(I)]$ & $R_{1}=0.0865, \mathrm{w} R_{2}=0.2132$ \\
\hline Final $R$ indexes [all data] & $R_{1}=0.1184, \mathrm{w} R_{2}=0.2290$ \\
\hline Largest diff. peak/hole $/\left(e^{\bullet} \AA^{-3}\right)$ & $1.53 /-0.89$ \\
\hline
\end{tabular}

和 DISQ 内存在分子内 $\mathrm{N}-\mathrm{H} \cdots \mathrm{O}$ 作用力, 且分子 $\mathbf{S Q}$ 和 DISQ 的二面角为 $0^{\circ}$, 分子 MeSQ 和 DIMeSQ 的二面角 分别为 $1^{\circ}$ 和 $13^{\circ}$, 这与紫外 - 可见吸收光谱和荧光发射光 谱结果一致, 由于分子内 $\mathrm{N}-\mathrm{H} \cdots \mathrm{O}$ 作用, 增加了分子 平面性, 促进了吸收和发射光谱红移. 再根据前线分子 轨道分布图(图 8), 这 4 个分子的 HOMO 轨道和 LUMO 轨道存在着明显的电子云重叠, 因此具有电子耦合能 力, 这也与紫外-可见吸收光谱和苂光发射光谱结果一 致. 进一步证明了, 光激发的过程中, 分子结构的变化 是可以忽略的.

\section{2 结论}

在本工作中, 通过多步合成的方法, 合成了 4 个不 同取代吲哚修饰的方酸菁衍生物. 方酸菁衍生物是通过 在方酸母核 1,3-位引入吲哚结构, 并在吲哚结构上引入 不同的碘取代、 $N$-氢取代或 $N$-甲基取代，得到四个 D-A-D 构型的染料, 化合物结构通过 ${ }^{1} \mathrm{H}$ NMR, ${ }^{13} \mathrm{C}$ NMR 和 MS 等进行了表征. 研究发现, 这 4 种化合物都具有 强的近红外吸收性，且碘取代分子由于重原子效应，增

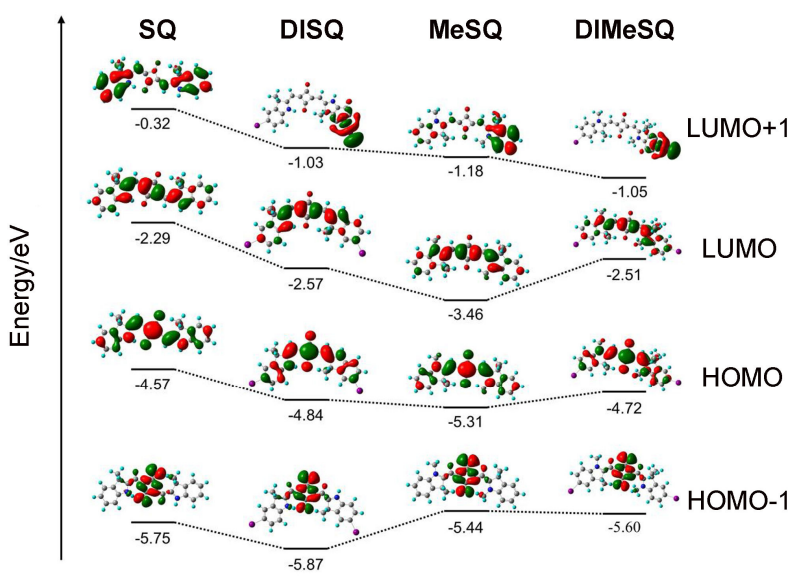

图 $8 \mathrm{SQ} 、$ DISQ、MeSQ 和 DIMeSQ 前线分子轨道分布图 Figure 8 Selected frontier molecular orbitals of SQ、DISQ、 MeSQ and DIMeSQ

强了光敏剂自旋-轨道耦合作用, 从而增大 $\mathrm{S}_{0} \rightarrow \mathrm{T}_{1}$ 吸收 跃迁和 $\mathrm{S}_{1} \rightarrow \mathrm{T}_{1}$ 体系间䇀跃的几率, 即增加了 $\mathrm{T}_{1}$ 态粒子 的布局数，有利于促进分子系间窝跃; $N$-氢取代分子由 于分子内 $\mathrm{N}-\mathrm{H} \cdots \mathrm{O}$ 作用力存在, 有益于稳定分子中心 受体结构, 造成电荷分离程度减小, 因而 $\pi \rightarrow \pi *$ 跃迁能 级降低，使其吸收带的最大吸收波长向长波方向移动， 有利于 $S_{1}$ 态的形成，从而促进了分子的系间鼌跃. 因 此，具有碘取代和 $\mathrm{N}-\mathrm{H}$ 取代协同作用的分子DISQ，表 现出良好的光物理化学性质, 具有多样性的应用潜能, 如可被应用于光动力治疗光敏剂等.

\section{3 实验部分}

\section{1 仪器与试剂}

AVANCE-400 型核磁共振波谱仪(德国 Bruker 公 司); TSQ Endura 超高效液相色谱串联三重四极杆质谱 联用仪(美国 Thermo Fisher 公司); UV-2700 紫外可见分 光光度计(日本岛津有限公司); FLS980 稳态瞬态荧光光 谱仪(英国 Edinburgh 公司); 万分之一电子天平(上海天 美天平仪器有限公司).

实验药品: 4-碘苯胺、苯肼、亚硝酸钠、二水氯化 亚锡、3-甲基-2-丁酮、碘甲烷、3,4-二羟基-3-环丁烯-1,2二酮、1,3-二苯基异苯并呋喃、亚甲基蓝、氢氧化钠、 无水硫酸钠(分析纯，上海安耐吉化学技术有限公司). 实验溶剂: 盐酸、冰乙酸、正丁醇、甲苯、乙醚、石油 醚、乙酸乙酯、二氯甲烷(分析纯, 天津市大茂化学试剂 厂).

\section{2 方酸菁衍生物及其中间体的合成方法}

中间体 $2 \mathbf{b}$ 按照文献[27]中的方法合成: 将 4-碘苯胺

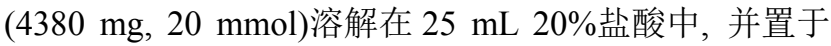
$150 \mathrm{~mL}$ 圆底烧瓶, 冷却至 $0{ }^{\circ} \mathrm{C}$. 同时称取亚硝酸钠 
(1448 mg, $21 \mathrm{mmol}$ )溶解在 $30 \mathrm{~mL}$ 水中, 将亚硝酸钠水 溶液于 $20 \mathrm{~min}$ 内缓慢滴加入上述反应液中, 将该反应液 于 $0{ }^{\circ} \mathrm{C}$ 下搅拌 $1.5 \mathrm{~h}$ 后. 称取二水氯化亚锡 $(11282 \mathrm{mg}$, $50 \mathrm{mmol}$ )溶解在 $15 \mathrm{~mL}$ 冰浓盐酸中, 并在 $5 \mathrm{~min}$ 内滴加 入反应液中, 将得到的悬浮液于 $0{ }^{\circ} \mathrm{C}$ 下继续摚拌 $1 \mathrm{~h}$. 反应结束后, 将浅棕色沉淀物过滤并用水洗涤三次, 然 后将该固体粗产物溶于二氯甲烷中, 以氢氧化钠水溶液 洗涤, 无水硫酸钠干燥, 过滤并旋干. 所得到的粗产物 利用柱层析提纯, 淋洗剂为 $[V$ (乙酸乙酯) : $V$ (石油醚 $)=$ $1: 10$ ], 得到 $2 \mathbf{b}$ 棕色粉末 $3100 \mathrm{mg}$, 产率 66\%. m.p. $104.6 \sim 108.8{ }^{\circ} \mathrm{C} ;{ }^{1} \mathrm{H}$ NMR $\left(400 \mathrm{MHz}, \mathrm{CDCl}_{3}\right) \delta: 7.48(\mathrm{~d}$, $J=8.9 \mathrm{~Hz}, 2 \mathrm{H}), 6.61(\mathrm{~d}, J=8.9 \mathrm{~Hz}, 2 \mathrm{H}), 3.38(\mathrm{~s}, 3 \mathrm{H}) ;{ }^{13} \mathrm{C}$ $\operatorname{NMR}\left(100 \mathrm{MHz}, \mathrm{CDCl}_{3}\right) \delta: 150.82,137.77,114.33,80.31$.

中间体 3a 按照文献[28]中的方法合成: 在氮气氛围 下, 将苯肼(8002 mg, $74 \mathrm{mmol}$ )和 3-甲基-2-丁酮(7648 $\mathrm{mg}, 88 \mathrm{mmol}$ )溶解在 $40 \mathrm{~mL}$ 冰乙酸中, $120{ }^{\circ} \mathrm{C}$ 下搅拌 $3 \mathrm{~h}$. 反应结束后冷却至室温, 真空除去溶剂, 得到深红色油 状液体粗产物. 将粗产物溶于乙酸乙酯中, 以水洗涤, 无水硫酸钠干燥, 过滤并旋干. 所得到的粗产物利用柱 层析提纯, 淋洗剂为 $[V$ (乙酸乙酯) $: V$ (石油 醚) $=1: 5]$, 得到 3a 浅黄色油状物 $8288 \mathrm{mg}$, 产率 70\%. ${ }^{1} \mathrm{H}$ NMR (400 $\left.\mathrm{MHz}, \mathrm{CDCl}_{3}\right) \delta: 7.49(\mathrm{~d}, J=7.6 \mathrm{~Hz}, 1 \mathrm{H}), 7.25 \sim 7.21(\mathrm{~m}$, 2H), $7.14(\mathrm{t}, J=7.4 \mathrm{~Hz}, 1 \mathrm{H}), 2.24$ (s, 3H), 1.24 (d, $J=0.8$ $\mathrm{Hz}, 6 \mathrm{H}) ;{ }^{13} \mathrm{C}$ NMR $\left(100 \mathrm{MHz}, \mathrm{CDCl}_{3}\right) \delta: 188.15,153.48$, $145.57,127.60,125.16,121.30,119.86,53.60,23.09$, 15.37 .

中间体 3b 按照文献[28]中的方法合成: 与合成 $\mathbf{3 a}$ 的方法相同, 浅黄色油状物 $1118 \mathrm{mg}$, 产率 $79 \% .{ }^{1} \mathrm{H}$ NMR (400 MHz, $\left.\mathrm{CDCl}_{3}\right) \delta: 7.56 \sim 7.49(\mathrm{~m}, 2 \mathrm{H}), 7.20(\mathrm{~d}$, $J=7.9 \mathrm{~Hz}, 1 \mathrm{H}), 2.16$ (s, 3H), 1.18 (s, 6H); ${ }^{13} \mathrm{C}$ NMR $(100$ $\left.\mathrm{MHz}, \mathrm{CDCl}_{3}\right) \delta: 188.37,153.21,148.10,136.64,130.62$, $121.77,90.04,53.99,22.91,15.33$.

中间体 4a 按照文献[28]中的方法合成 ${ }^{[28]}$ : 将中间体 3a (796 mg, $5.0 \mathrm{mmol})$ 和碘甲烷 $(851 \mathrm{mg}, 6.0 \mathrm{mmol})$ 置于 装有磁力摚拌棒的 $50 \mathrm{~mL}$ 微波反应容器中, 固定好反应 容器, 并在 $135{ }^{\circ} \mathrm{C}$ 下微波辐射 $20 \mathrm{~min}$. 反应结束后, 将 粗产物用乙梄洗涤三次, 过滤并干燥, 所需产物不需要 进一步纯化, 得到 4a 浅粉红色固体 $686 \mathrm{mg}$, 产率 48\%. m.p. $258.8 \sim 262.6{ }^{\circ} \mathrm{C}$.

中间体 $4 \mathrm{~b}$ 的合成：用类似于合成 $4 \mathrm{a}$ 的方法合成 $4 \mathrm{~b}$, 得到 4b 红棕色固体 $858 \mathrm{mg}$, 产率 57\%. m.p. 248.6 $253.0{ }^{\circ} \mathrm{C}$.

方酸衍生物 SQ 的合成: 在氮气氛下, 将方酸 $(114$ $\mathrm{mg}, 1.0 \mathrm{mmol}$ )置于装有 Dean-Stark 分水器的双颈烧瓶 中, 并溶解在 $10 \mathrm{~mL}$ 正丁醇和 $10 \mathrm{~mL}$ 甲苯的混合溶液中,
将反应液于 $115{ }^{\circ} \mathrm{C}$ 下搅拌 $3 \mathrm{~h}$ 后. 称取中间体 $3 \mathrm{a}(602$ $\mathrm{mg}, 2.0 \mathrm{mmol}$ )溶解在 $2 \mathrm{~mL}$ 正丁醇和 $6 \mathrm{~mL}$ 甲苯的混合溶 液中, 并逐滴加入至上述反应液中, $115{ }^{\circ} \mathrm{C}$ 下继续摚拌 $4 \mathrm{~h}$. 反应结束后冷却至室温, 减压旋转蒸发除去溶剂. 将得到的粗产物利用柱层析提纯, 淋洗剂为 $[V($ 乙酸乙 酯)： $V$ (石油醚) $=1: 1$ ], 得到 $\mathbf{S Q}$ 褐色固体粉末 $206 \mathrm{mg}$, 产率 52\%. m.p. 278.6 282.6 ${ }^{\circ} \mathrm{C} ;{ }^{1} \mathrm{H}$ NMR $(400 \mathrm{MHz}$, $\left.\mathrm{CDCl}_{3}\right) \delta: 12.68(\mathrm{~d}, J=119.0 \mathrm{~Hz}, 2 \mathrm{H}), 7.31 \sim 7.24(\mathrm{~m}$, 4H), $7.21 \sim 7.05$ (m, 4H), 5.49 (d, $J=29.4 \mathrm{~Hz}, 2 \mathrm{H}), 1.45$ (s, 12H); ${ }^{13} \mathrm{C}$ NMR $\left(100 \mathrm{MHz}, \mathrm{CDCl}_{3}\right) \delta: 183.41,175.75$, $175.04,141.92,138.99,128.28,123.20,122.47,122.35$, 111.50, 85.93, 48.70, 26.64; HRMS (ESI) calcd for $\mathrm{C}_{26} \mathrm{H}_{24} \mathrm{~N}_{2} \mathrm{O}_{2}[\mathrm{M}+\mathrm{H}]^{+}$397.1903, found 397.1838.

方酸衍生物 DISQ 的合成: 用类似于合成 SQ 的方 法合成, 得到绿色固体粉末 $446 \mathrm{mg}$, 产率 $69 \%$. m.p. $267.6 \sim 270.6{ }^{\circ} \mathrm{C} ;{ }^{1} \mathrm{H}$ NMR $\left(400 \mathrm{MHz}, \mathrm{CDCl}_{3}\right) \delta: 12.72(\mathrm{~d}$, $J=128.9 \mathrm{~Hz}, 2 \mathrm{H}), 7.63 \sim 7.49(\mathrm{~m}, 4 \mathrm{H}), 6.92(\mathrm{dd}, J=19.9$, $8.1 \mathrm{~Hz}, 2 \mathrm{H}), 5.48(\mathrm{~d}, J=33.3 \mathrm{~Hz}, 2 \mathrm{H}), 1.43(\mathrm{~s}, 12 \mathrm{H}) ;{ }^{13} \mathrm{C}$ NMR $\left(100 \mathrm{MHz}, \mathrm{CDCl}_{3}\right) \delta: 183.26,175.70,175.09$, $141.59,141.48,137.18,131.53,113.37,113.17,86.50$, 86.09, 53.45, 48.75, 26.61; HRMS (ESI) calcd for $\mathrm{C}_{26} \mathrm{H}_{22}-$ $\mathrm{I}_{2} \mathrm{~N}_{2} \mathrm{O}_{2}[\mathrm{M}+\mathrm{H}]^{+}$647.9771, found 647.9836.

方酸衍生物 MeSQ 的合成: 用类似于合成 SQ 的方 法合成, 得到蓝色固体粉末 $219 \mathrm{mg}$, 产率 76\%. m.p. $298.5 \sim 302.2{ }^{\circ} \mathrm{C}$; ${ }^{1} \mathrm{H}$ NMR $\left(400 \mathrm{MHz}, \mathrm{CDCl}_{3}\right) \delta: 7.32(\mathrm{dd}$, $J=16.6,7.4 \mathrm{~Hz}, 4 \mathrm{H}), 7.14$ (d, $J=6.9 \mathrm{~Hz}, 2 \mathrm{H}), 6.99$ (d, $J=$ $7.4 \mathrm{~Hz}, 2 \mathrm{H}), 5.92(\mathrm{~s}, 2 \mathrm{H}), 3.57$ (s, 6H), $1.76(\mathrm{~s}, 12 \mathrm{H}) ;{ }^{13} \mathrm{C}$ NMR $\left(100 \mathrm{MHz}, \mathrm{CDCl}_{3}\right) \delta: 179.38,170.99,143.04$, 127.85, 123.87, 122.25, 109.27, 86.82, 86.78, 49.26, 27.05; HRMS (ESI) calcd for $\mathrm{C}_{28} \mathrm{H}_{28} \mathrm{~N}_{2} \mathrm{O}_{2}[\mathrm{M}+\mathrm{H}]^{+}$425.2216, found 425.2151 .

方酸衍生物 DIMeSQ 的合成: 用类似于合成 SQ 的 方法合成, 得到红棕色固体粉末 $219 \mathrm{mg}$, 产率 $36 \%$. m.p. $287.3 \sim 292.2{ }^{\circ} \mathrm{C}$; ${ }^{1} \mathrm{H}$ NMR $\left(400 \mathrm{MHz}, \mathrm{CDCl}_{3}\right) \delta$ : $7.69 \sim 7.61(\mathrm{~m}, 4 \mathrm{H}), 6.83(\mathrm{~d}, J=8.3 \mathrm{~Hz}, 2 \mathrm{H}), 6.27(\mathrm{~s}, 2 \mathrm{H})$, $3.63(\mathrm{~s}, 6 \mathrm{H}), 1.73(\mathrm{~s}, 12 \mathrm{H}) ;{ }^{13} \mathrm{C} \mathrm{NMR}\left(100 \mathrm{MHz}, \mathrm{CDCl}_{3}\right) \delta$ : $179.23,170.94,142.71,132.77,130.34,129.58,100.17$, 86.25, 85.84, 54.01, 49.31, 27.02; HRMS (ESI) calcd for $\mathrm{C}_{28} \mathrm{H}_{26} \mathrm{I}_{2} \mathrm{~N}_{2} \mathrm{O}_{2}[\mathrm{M}+\mathrm{H}]^{+}$677.0103, found 677.0084.

\section{3 光谱测试方法}

将待测物 SQ、DISQ、MeSQ 和 DIMeSQ 配制成 $1.0 \times 10^{-3} \mathrm{~mol} \cdot \mathrm{L}^{-1}$ 溶液, 溶剂为二氯甲烷. 测试浓度一 般为 $1.0 \times 10^{-5} \mathrm{~mol} \cdot \mathrm{L}^{-1}$, 具体见标注.

紫外-可见吸收光谱测定：首先将装有溶剂的双通 比色具放入 UV2700 光谱仪中, 进行空白测试, 再对化 
合物进行测试. 苂光发射光谱测定: 将装有溶液的四通 比色皿放入 FLS980 稳态瞬态光谱仪中, 选择合适的激 发波长和狭缝，进行测试.

苂光寿命测定: 将装有溶液的四通比色皿放入 FLS980 稳态瞬态光谱仪中，选择合适的测试范围、激发 波长和狭缝, 进行测试.

荧光量子产率的测定: 根据紫外可见吸收光谱的吸 光度 $\mathrm{A}$ 来调整浓度, 使测试溶液在激发波长处的吸光度 在 0.1 左右. 首先将装有溶剂的比色皿放入 FLS980 稳态 瞬态光谱仪的积分球内, 选择合适的激发波长, 得到空 白的光谱图. 然后将装有溶液的比色㿼置于积分球内, 直接进行测试得到溶液的光谱图. 利用机器自带的软件 处理数据得到溶液的绝对苂光量子产率.

单线态氧量子产率的测定: 以 1,3-二苯基异苯并呋 喃作为单线态氧清除剂, 根据紫外一可见吸收光谱的吸 光度 $A$ 来调整浓度, 使测试溶液在激发波长处的吸光度 在 0.1 左右, 清除剂 1,3-二苯基异苯并呋喃 $415 \mathrm{~nm}$ 处吸 光度在 1.0 左右, 利用激发波长光照射样品, 在不同照 射时间下测定紫外-可见吸收光谱, 根据 $415 \mathrm{~nm}$ 处吸光 度的变化来计算单线态氧量子产率, 具体通过公式(1) 计算, 以亚甲基蓝作为标准物 $\left(\Phi_{\Delta}=0.57\right.$, 二氯甲烷).

$$
\Phi_{\text {sam }}=\Phi_{\text {std }}\left(\frac{1-10^{-A_{\text {std }}}}{1-10^{-A_{\text {sam }}}}\right)\left(\frac{k_{\text {sam }}}{k_{\text {std }}}\right)\left(\frac{\eta_{\text {sam }}}{\eta_{\text {std }}}\right)
$$

其中 “sam” 和 “std” 分别代表待测样品和标准品. $\Phi, A$, $k$ 和 $\eta$ 分别代表单线态氧量子产率, 激发波长处的吸光 度，1,3-二苯基异苯并呋喃的吸光度随时间变化的斜率 以及测量溶剂的折射率.

\section{4 晶体结构测试方法}

以二氯甲烷-正己烷为溶剂, 配制适量化合物 DISQ 的饱和溶液, 在室温下缓慢挥发, 约两周后析出 绿色单晶用于结构测定. 晶体的 X 射线数据是在室温下 由 Bruker SMART $1000 \mathrm{CCD}$ 衍射仪收集, 数据处理使 用 SAINT+程序包, 对 1958 个 $[I>2 \sigma(I)]$ 的可观察点用 直接法解析, 用全矩阵最小二乘法对 220 个参数进行修 正. 对非氢原子采用各向异性热参数, 对氢原子采用各 向同性热参数.

\section{5 理论计算方法}

本工作计算结果使用了 Gaussian 09W 软件, 基态 的计算使用了密度泛函理论(DFT)方法对分子 $\mathbf{S Q}$ 、

DISQ、MeSQ 和 DIMeSQ 进行计算. 计算采用混合泛 函 B3LYP 方法，分子 SQ 和分子 MeSQ 采用 6-31G (d,p) 基组, 分子 DISQ 和分子 DIMeSQ 采用 GENECP 基组, 其中重元素 I 使用了 LANL2DZ 基组, C、H、O、 $\mathrm{N}$ 等 小原子使用了 6-31G $(\mathrm{d}, \mathrm{p})$ 基组.
辅助材料(Supporting Information) 中间体及其最终 产物表征谱图、高斯计算数据. 这些材料可以免费从本 刊网站(http://sioc-journal.cn/)上下载.

\section{References}

[1] Yao, Y.; Liang, Y.; Shrotriya, V.; Xiao, S.; Yang, Y. Adv. Mater. 2007, 19, 3979.

[2] Gao, F.; Feng, X.; Tham, H. P.; Zhang, R.; Zhang, Y.; Liu, S.; Zhao, L.; Zheng, Y.; Zhao, Y. Small 2016, 12, 5239.

[3] Liu, C. H.; Li, Y. H.; Qi, F. P.; Long, L. P.; Yang, R. H. Sci. Sin. Chim. 2017, 47, 1015 (in Chinese) (刘长辉, 李银辉, 齐风佩, 龙立平, 杨荣华, 中国科学: 化学, 2017, 47, 1015.)

[4] Wu, B.; Li, Y.; Zhan, C.; Zeng, F.; Wu, S. Anal. Chem. 2018, 90, 9359.

[5] Atchison, J.; Kamila, S.; Nesbitt, H.; Logan, K. A.; Nicholas, D. M.; Fowley, C.; Davis, J.; Callan, B.; McHale, A. P.; Callan, J. F. Chem. Commun. 2017, 53, 2009.

[6] Jiao, L.; Song, F.; Cui, J.; Peng, X. Chem. Commun. 2018, 54, 9198.

[7] Li, L.; Chen, W.; Tian, Y.; Chen, H.; Yin, J. Chin. Chem. Lett. 2019, 30, 1689.

[8] Shi, C. H.; Wu, J. B.; Pan, D. F. J. Biomed. Opt. 2016, 21, 50901.

[9] Yoon, H. Y.; Koo, H.; Choi, K. Y.; Lee, S. J.; Kim, K.; Kwon, I. C.; Leary, J. F.; Park, K.; Yuk, S. H.; Park, J. H.; Choi, K. Biomaterials 2012, 33, 3980.

[10] Chinnathambi, S.; Shirahata, N. Sci. Technol. Adv. Mater. 2019, 20, 337.

[11] Tian, Y.; Zheng, L.; Lu, G. M. J. Clin. Radiol. 2012, 31, 897 (in Chinese).

(田迎，郑玲，卢光明，临床放射学杂志，2012, 31, 897.)

[12] Bruijns, B. B.; Tiggelaar, R. M.; Gardeniers, J. G. E. Anal. Biochem. 2016, 511, 74 .

[13] Rapozzi, V.; Beverina, L.; Salice, P.; Pagani, G. A.; Camerin, M.; Xodo, L. E. J. Med. Chem. 2010, 53, 2188.

[14] Lim, S. H.; Thivierge, C.; Nowak-Sliwinska, P.; Han, J.; Bergh, H.; Wagnieres, G.; Burgess, K.; Lee, H. B. J. Med. Chem. 2010, 53, 2865.

[15] Saha, P. C.; Chatterjee, T.; Pattanayak, R.; Das, R. S.; Mukherjee, A.; Bhattacharyya, M.; Guha, S. ACS Omega 2019, 4, 14579.

[16] Obata, M.; Hirohara, S.; Tanaka, R.; Kinoshita, I.; Ohkubo, K.; Fukuzumi, S.; Tanihara, M.; Yano, S. J. Med. Chem. 2009, 52, 2747.

[17] Fu, P.; Zhang, C.; Zhang, W. D.; Liu, R. H.; Xu, X. K. J. Pharm. Pract. 2005, (1), 6 (in Chinese).

(傅范, 张川, 张卫东, 柳润辉, 徐希科, 药学实践杂志, 2005, (1), 6.)

[18] Schneeweis, A. P. W.; Hauer, S. T.; Reiss, G. J.; Müller, T. J. J. Chem.-Eur. J. 2019, 25, 3582

[19] Huang, L.; Li, Z.; Zhao, Y.; Yang, J.; Yang, Y.; Pendharkar, A. I.; Zhang, Y.; Kelmar, S.; Chen, L.; Wu, W.; Zhao, J.; Han, G. $A d v$. Mater. 2017, 29, 1604789.

[20] Wang, Z.; Zhao, J. Org. Lett. 2017, 19, 4492.

[21] Hussain, M.; El-Zohry, A. M.; Gobeze, H. B.; Zhao, J.; D’Souza, F.; Mohammed, O. F. J. Phys. Chem. A 2008, 122, 6081.

[22] Kandrashkin, Y. E.; Wang, Z.; Sukhanov, A. A.; Hou, Y.; Zhang, X.; Liu, Y.; Voronkova, V. K.; Zhao, J. J. Phys. Chem. Lett. 2019, 10, 4157.

[23] Cohen, M.; Turnbull, D. J. Chem. Phys. 1959, 5, 1164.

[24] Sreejith, S.; Carol, P.; Chithra, P.; Ajayaghosh, A. J. Med. Chem. 2008, 18, 264. 
[25] Li, C. L.; Wang, L. Y.; Sun, G. F.; Zhang, Z. X. Chin. J. Org. Chem. 2006, 26, 442 (in Chinese).

(李春兰, 王兰英, 孙国峰, 张祖训, 有机化学, 2006, 26, 442.)

[26] Zhang, D. J.; Chu, Z. Y.; Xing, X.; Cheng, H. F. Mater. Rep. 2010, 11,74 (in Chinese).

(张东玖，楚增勇，邢欣，程海峰，材料导报, 2010, 11, 74.)

[27] Jordan, A.; Sukanta, K.; Heather, N.; Kieran, A. L.; Dean, M. N.; Colin, F.; James, D.; Bridgeen, C.; Anthony, P. M.; John, F. C.
Chem. Commun. 2017, 53, 2009

[28] Serpe, L.; Ellena, S.; Barbero, N.; Foglietta, F.; Prandini, F.; Gallo, M. P.; Levi, R.; Barolo, C.; Canaparo, R.; Visentin, S. Eur. J. Med. Chem. 2016, 113, 187.

[29] Danaboyina, R.; Abraham, J.; Nese, C.; Nadukkudy, V.; Suresh, D.; Manapurathu, V. Photochem. Photobiol. 1997, 65, 783.

(Fan, Y.) 